\title{
Perturba e Observa Adaptável para Elevar a Eficiência de Sistemas Fotovoltaicos *
}

\author{
Cleiton O. de Souza* Tiago J. M. Dezuo* \\ * Departamento de Engenharia Elétrica, Universidade do Estado de \\ Santa Catarina, Joinville/SC, (e-mails: cleitonolivsouza@gmail.com, \\ tiago.dezuo@udesc.br).
}

\begin{abstract}
This paper presents a proposal for a Maximum Power Point Tracking (MPPT) algorithm, of the Perturb and Observe ( $\mathrm{P} \& \mathrm{O})$ type, adaptable to increase the efficiency of photovoltaic generation. As a by-product, a methodology for analyzing the efficiency of a photovoltaic system, considering both its transient nature and its regime condition is also presented. This analysis, based on the energy lost (available but not generated) by an MPPT strategy, allows the evaluation of the transient efficiency of a system, assisting in comparisons between different strategies. From this analysis, in particular for a $\mathrm{P} \& \mathrm{O}$ technique, one perceives the existence of a compromise between an efficient transient and a steady state without waste of energy. Motivated by the issue in the traditional $\mathrm{P} \& \mathrm{O}$, the proposed adaptive algorithm has the goal of, through the method of imposing increments of adaptable amplitudes at each MPPT step, making both transient and steady state more efficient. Two numerical examples illustrate the efficiency analysis and the application of the proposed algorithm.

Resumo: Este artigo apresenta uma proposta de algoritmo de rastreamento do ponto de máxima potência (MPPT), do tipo Perturba e Observa ( $\mathrm{P} \& \mathrm{O})$, adaptável para elevar a eficiência da geração fotovoltaica. Como um sub-produto, tem-se uma metodologia de análise da eficiência de um sistema fotovoltaicos, considerando tanto sua condição transitória de rastreamento quanto sua condição de regime. Esta análise, baseada em estimar a energia perdida (não aproveitada) por uma estratégia de MPPT, permite avaliar a eficiência transitória de um sistema, auxiliando na comparação entre diferentes estratégias. A partir de tal análise, em particular para a técnica $\mathrm{P} \& \mathrm{O}$, percebe-se a existência de um compromisso entre um transitório eficiente e um regime sem desperdícios de energia. Motivado pela problemática do $\mathrm{P} \& \mathrm{O}$ tradicional, o algoritmo adaptável proposto visa mitigar este compromisso através da imposição de incrementos de amplitude adaptável a cada passo do MPPT, realizando tanto transitórios quanto regimes mais eficientes. Dois exemplos numéricos ilustram a análise de eficiência e aplicação do algoritmo proposto.
\end{abstract}

Keywords: Photovoltaic systems; MPPT; P\&O; Efficiency; Adaptative algorthm.

Palavras-chaves: Sistemas fotovoltaicos; MPPT; P\&O; Eficiência; Algoritmo adaptativo.

\section{INTRODUÇÃO}

O interesse mundial em fontes de energia sustentáveis está aumentando devido à conscientização ambiental e ao aumento dos preços do petróleo. Na verdade, o custo da eletricidade fotovoltaica (PV), tecnologia de conversão da energia solar diretamente em energia elétrica, diminuiu drasticamente na última década (European Photovoltaic Industry Association (EPIA), 2018) e a paridade com o varejo de eletricidade e combustíveis à base de petróleo vem sendo alcançado em muitos países de todo o mundo (Di Francia, 2015). O elevado crescimento populacional e o desenvolvimento tecnológico, que acarretam no aumento da demanda de energia, bem como a redução de custos e a popularização são também fatores determinantes ao expressivo desenvolvimento dos sistemas PV.

\footnotetext{
* Trabalho realizado com apoio da FAPESC através do Edital No 04/2018 e do FNDE através do Programa de Educação Tutorial da $\mathrm{SESu} / \mathrm{MEC}$.
}

A Figura 1, divulgada pela European Photovoltaic Industry Association (EPIA) (2018), apresenta a capacidade instalada de sistemas fotovoltaicos em nível mundial entre os anos de 2000 e 2017. Destaca-se que o crescimento apresenta comportamento aproximadamente exponencial, atingindo uma capacidade instalada de cerca de 404.5 GW em 2017. Entretanto, uma questão em aberto é: o quanto se deixa de produzir dessa energia disponível devido a métodos ineficientes de controle da geração?

Neste contexto, existem diversas formas práticas de se obter o rastreamento do ponto máximo de potência $\left(\mathrm{MPPT}^{1}\right)$ de um arranjo fotovoltaico, o que indica a importância deste tratamento nos sistemas. Os vários métodos existentes se diferem em características como complexidade, custo, eficiência, implementação, sistema de hardware e em outros aspectos. De maneira geral, as técnicas de MPPT têm o objetivo de determinar constantemente, de forma automática, os valores de tensão ou

1 Do inglês, Maximum Power Point Tracking. 


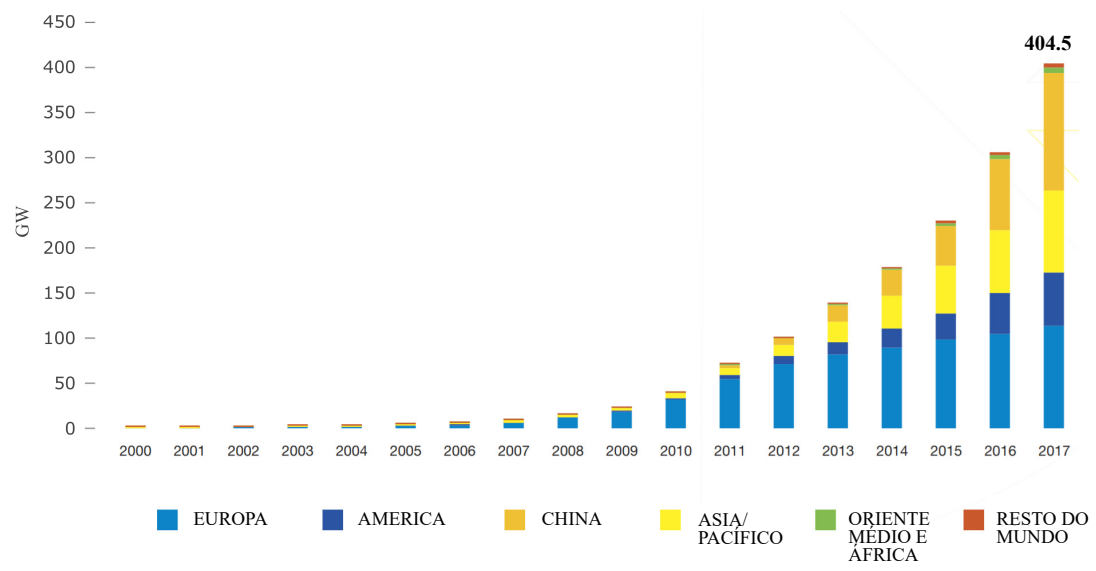

Figura 1. Evolução da capacidade instalada global.

corrente adequados para a extração da máxima potência do arranjo, visto que os valores ótimos para este ajuste sofrem influência de variáveis não controladas, como a temperatura e a irradiação (Soualmia and Chenni, 2016). Uma lista detalhada de mais de 40 métodos de MPPT pode ser encontrada em (Soualmia and Chenni, 2016).

Dentre as estratégias MPPT, uma das mais conhecidas e utilizadas, devido principalmente a sua simplicidade de implementação, é a conhecida como Perturba e Observa $(\mathrm{P} \& \mathrm{O})$. Este método tem como base a forma típica (concava) da curva característica de potência do arranjo PV em função das variáveis de tensão ou de corrente. Sua operação se dá através do deslocamento forçado do ponto de operação em uma direção (perturbar) e observar o resultado na potência de saída do gerador fotovoltaico. Quando há um aumento da potência, o algoritmo atua de forma a continuar perturbando o sistema na mesma direção e, caso contrário, atua na direção oposta. Entretanto, existe um compromisso entre um $\mathrm{P} \& \mathrm{O}$ rápido e um $\mathrm{P} \& \mathrm{O}$ preciso, levando a um dilema sobre qual ajuste da técnica resulta na melhor eficiência.

Este artigo apresenta uma proposta de algoritmo MPPT adaptável para elevar a eficiência da geração fotovoltaica. Como um sub-produto, tem-se uma metodologia de análise da eficiência de um sistema fotovoltaicos, considerando tanto sua condição transitória de rastreamento quanto sua condição de regime. Esta análise, baseada em estimar a energia perdida (não aproveitada) por uma estratégia de MPPT, permite avaliar a eficiência transitória de um sistema, auxiliando na comparação entre diferentes estratégias. A partir de tal análise, em particular para a técnica $\mathrm{P} \& \mathrm{O}$, percebe-se a existência de um compromisso entre um transitório eficiente e um regime sem desperdícios de energia. Motivado pela problemática do $\mathrm{P} \& \mathrm{O}$ tradicional, o algoritmo adaptável proposto visa mitigar este compromisso através da imposição de incrementos de amplitude adaptável a cada passo do MPPT, realizando transitórios e regimes mais eficientes.

A organização do documento é apresentada a seguir. Esta seção termina com a pré-definição de símbolos usados no artigo. A próxima seção apresenta o modelo de sistemas fotovoltaicos e outros conceitos básicos. Os algoritmos MPPT, com detalhe ao P\&O, bem como a metodologia de análise são apresentadas na Seção 3. A Seção 4 é dedicada ao desenvolvimento do algoritmo proposto. Simulações numéricas ilustram os resultados da nova estratégia na Seção 5 e algumas conclusões finalizam o artigo.

Os seguintes símbolos serão usados no decorrer deste documento.

Símbolos. $R_{s}$ - Resistência série do módulo; $R_{p}$ - Resistência paralelo do módulo; $\epsilon$ - Carga do elétron $\left(1.6 \times 10^{-19}\right.$ C); $\eta$ - Fator de qualidade da junção p-n; $\kappa$ - Constante de Boltzmann $\left(1.38 \times 10^{-23} \mathrm{~J} / \mathrm{K}\right) ; T_{r}$ - Temperatura de referência (STC) $\left(298 \mathrm{~K}\right.$, ou seja, $\left.25^{\circ} \mathrm{C}\right) ; G_{r}$ - Intensidade de irradiação de referência (STC) $\left(1000 \mathrm{~W} / \mathrm{m}^{2}\right) ; T$ - Temperatura da célula, em Kelvin; $G$ - Intensidade de irradiação solar recebida, em $\mathrm{W} / \mathrm{m}^{2} ; I_{s c}$ - Corrente de curto circuito do módulo; $V_{o c}$ - Tensão de circuito aberto do módulo; $\gamma$ - Coeficiente de temperatura de $I_{s c} ; E_{g}$ Energia de banda do silício $(1.1 \mathrm{eV}) ; V_{p v}$ - Tensão de saída nos terminais do arranjo fotovoltaico; $I_{p v}$ - Corrente de saída nos terminais do arranjo fotovoltaico; $M_{p}$ - Número de módulos em paralelo; $M_{s}$ - Número de módulos em série; $N_{s}$ - Número de células fotovoltaicas em série em cada módulo.

\section{SISTEMAS FOTOVOLTAICOS}

Um módulo fotovoltaico pode ser modelado como uma fonte de corrente controlada por tensão ou, alternativamente, como uma fonte de tensão controlada por corrente. Considerando um arranjo de $M_{p}$ fileiras em paralelo de $M_{s}$ módulos em série cuja temperatura é $T$ e recebe uma irradiação $G$, a tensão $V_{p v}$ e corrente $I_{p v}$ em seus terminais estão relacionadas através da função não linear (Casaro, 2009)

$$
\begin{aligned}
0=I_{p h}-(1 & \left.+\frac{R_{s}}{R_{p}}\right) \frac{I_{p v}}{M_{p}}-\frac{V_{p v}}{R_{p} N_{s} M_{s}} \\
& -I_{r}\left(\exp \left(\frac{\epsilon}{\eta \kappa T}\left(\frac{V_{p v}}{N_{s} M_{s}}+\frac{R_{s} I_{p v}}{M_{p}}\right)\right)-1\right)
\end{aligned}
$$

onde

$$
\begin{gathered}
I_{p h}=\left(I_{s c}+\gamma\left(T-T_{r}\right)\right) \frac{G}{G_{r}} \\
I_{r}=I_{r r}\left(\frac{T}{T_{r}}\right)^{3} \exp \left(\frac{\epsilon E_{g}}{\eta \kappa}\left(\frac{1}{T_{r}}-\frac{1}{T}\right)\right) \\
I_{r r}=\frac{I_{s c}-\frac{V_{o c}}{R_{p}}}{\exp \left(\frac{\epsilon V_{o c}}{\eta \kappa T_{r}}\right)-1} .
\end{gathered}
$$


Note em (1) que não é possível isolar $V_{p v}$ ou $I_{p v}$ para determinar seu valor algebricamente. Por este motivo, utiliza-se o método de Newton para encontrar as raízes da Equação (1), isto é, valores de $I_{p v}$ para cada respectivo $V_{p v}$ dado. Além disso, $T$ e $G$ são variáveis incertas.

Para uma dada distribuição uniforme de irradiação e temperatura sobre um arranjo $\mathrm{PV}$, a caraterística $I-V$ obtida da Equação (1) do arranjo tem a forma apresentada na Figura 2, onde nota-se que a potência $\left(P_{p v}=I_{p v} V_{p v}\right.$, ponto a ponto) possui um único ponto de máximo.

(a)

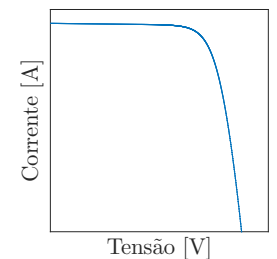

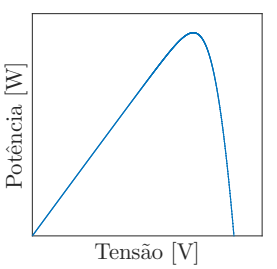

(b)

Figura 2. Curvas características (a) $I-V$ e (b) $P-V$ de um sistema sob condições uniformes.

Diversos fatores interferem na eficiência dos sistemas fotovoltaicos, dentre os quais destaca-se a influência dos parâmetros incertos $T$ e $G$ nas curvas características. A variação destes parâmetros tem como consequência a mudança nos valores de corrente e tensão de operação do arranjo que resulta no ponto de potência máxima (MPP). Com a curva $I-V$ desconhecida em tempo real, faz-se necessária alguma heurística para levar a operação ao MPP.

\section{RASTREAMENTO DO PONTO DE MÁXIMA POTÊNCIA}

As técnicas de rastreamento do máximo ponto de potência (MPPT) têm como intuito identificar o MPP, proporcionando uma maior eficiência ao sistema. Existem diversas formas práticas de se realizar o MPPT de um sistema PV, resultando em diferentes níveis de aproveitamento da geração, o que indica a importância de pesquisas nesta área. Os vários métodos existentes se diferem em características como complexidade, custo, eficiência, implementação, sistema de hardware, etc. (Soualmia and Chenni, 2016).

\subsection{Perturba e Observa}

A estratégia proposta neste artigo tem como base uma das técnicas mais aplicadas devido a sua simplicidade, a conhecida como Perturba e Observa (P\&O). Este método MPPT tem como funcionamento básico a análise da curva característica da potência da Figura 2(b) e pode apresentar como variáveis de ajuste a tensão $V_{p v}$ ou a corrente $I_{p v}$, observando o comportamento da potência gerada $P_{p v}$.

No algoritmo de $\mathrm{P} \& \mathrm{O}$ são comparados os valores da iteração atual com a iteração anterior. Quando há um aumento da potência na iteração atual, o algoritmo atua de forma a continuar aumentando a potência, repetindo o incremento (ou decremento) aplicado anteriormente na corrente ou a tensão. Mas se houver uma diminuição da potência, o algoritmo atua de forma a voltar a aumentar a potência e age no sentido contrário ao da última variação.

Para este trabalho, a variável de interesse para o controle é a corrente do arranjo. Entretanto, o funcionamento considerando a tensão pode ser executado de forma análoga.
O método $\mathrm{P} \& \mathrm{O}$ com a corrente como variável ajustada é ilustrado pelo Algoritmo 1.

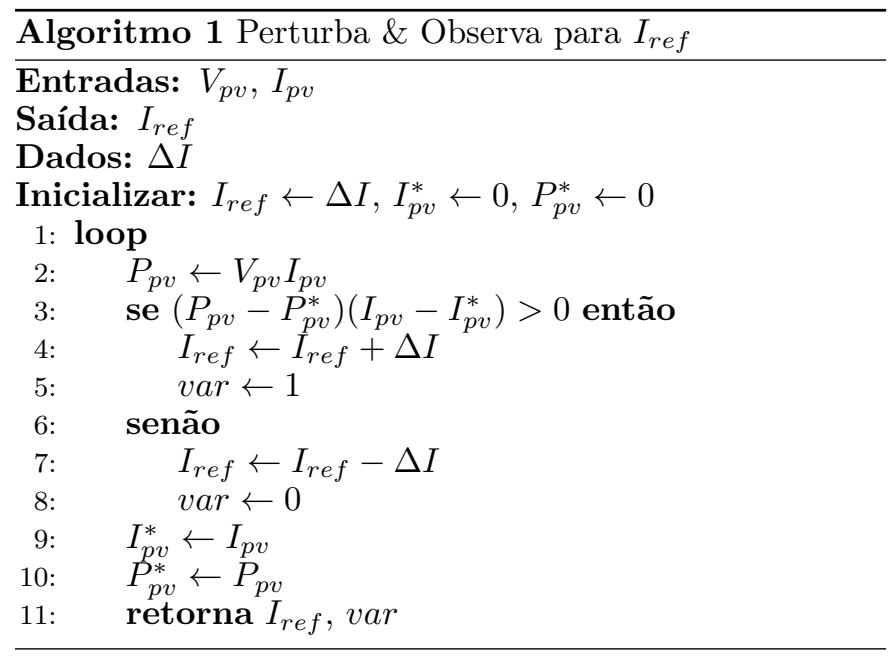

De acordo com o Algoritmo 1, é realizada a leitura dos valores de $V_{p v}$ e $I_{p v}$ em um dado momento para o cálculo de $P_{p v}$. Compara-se esta potência com a potência armazenada da iteração anterior $\left(P_{p v}^{*}\right)$. Caso estas sejam iguais, o algoritmo não faz nenhuma modificação. Caso sejam diferentes, verifica-se se a potência aumentou ou diminuiu e qual foi o sentido da última variação imposta à corrente. Este sentido de variação está sendo armazenado em uma variável denominada var no Algoritmo 1 a ser utilizada na técnica MPPT proposta neste artigo em seguida.

\subsection{Análise de Eficiência}

O conceito de eficiência de um sistema de geração pode ser definido como a razão entre a energia que este entrega de fato a sua carga e a energia total disponível em sua entrada. Em outras palavras, quanto mais perdas ocorrem no processo, menor a eficiência. Tais perdas podem ocorrer em diversos níveis, como: na conversão da energia solar (radiação) em energia elétrica, em um mau rastreamento do MPP, perdas elétricas, por comutação nos circuitos conversores, dentre outras causas menores.

Ao se analisar eficiência da convergência para o MPP, ou seja, o quanto da máxima energia elétrica que o painel pode gerar é realmente aproveitada, é interessante explorar a aplicação de técnicas de MPPT e a eficiência destas. Sabe-se que as técnicas de MPPT são heurísticas que apresentam uma dinâmica particular de convergência para o MPP. Assim, a geração de energia fica comprometida por dois motivos: (i) transitório operando fora do MPP e (ii) imprecisão da operação ao redor do MPPT em regime.

Técnicas que têm como característica transitórios rápidos e regime impreciso levam vantagem quando a análise é realizada para intervalos de tempo menores. De maneira análoga, técnicas de transitório lento e regime preciso têm vantagem para intervalos mais longos (mais tempo em regime). Sabendo que o ponto de transição entre transitório e regime varia, torna-se difícil escolher um intervalo que resulte em uma comparação justa. Assim, para uma análise mais efetiva, deve-se ponderar se o sistema em questão sofrerá muitos transitórios ou terá sua operação em uma condição mais de regime. 
Uma maneira de avaliar a eficiência da energia gerada pelo arranjo PV é observando-se a energia gerada acumulada durante um certo intervalo de tempo, dada por

$$
E_{\text {gerada }}=\int_{t_{1}}^{t_{2}} P_{p v} d t
$$

Entretanto, a analise da eficiência através da energia gerada possui algumas dificuldades, pois a energia gerada está sempre crescendo com o tempo, e a taxas diferentes para cada sistema. Por este motivo, neste trabalho propõese a análise da energia perdida, que tende a aproximar-se de um valor constante quanto mais próxima a operação estiver do MPP. O cálculo de $E_{\text {perdida }}$ é dado por

$$
E_{\text {perdida }}=\int_{t_{1}}^{t_{2}}\left(P_{\text {max }}-P_{p v}\right) d t .
$$

onde $P_{\max }$ representa a potência em MPP para uma dada condição de $T$ e $G$.

Nota 1. Vale ressaltar que a análise da energia perdida depende de se conhecer $P_{\max }$, o que a torna não aplicável na prática. Portanto esta análise é restrita a simulações computacionais onde se tem acesso à condição que está sendo imposta ao sistema.

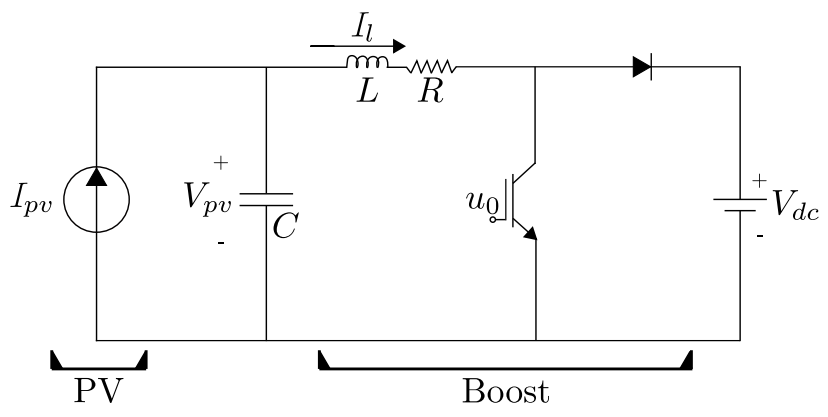

Figura 3. Topologia do sistema PV-Boost considerado.

Exemplo 1. (Eficiência do P\&O tradicional). Considere o sistema apresentado na Figura 3, extraído de (Dezuo et al., 2014), que consiste em um arranjo PV conectado a uma bateria através de um conversor Boost. São utilizadas as características de um módulo policristalino KC200GT do fabricante Kyocera, com 54 células interconectadas em série. Os dados deste módulo nas condições padrão de teste, em uma temperatura de $25{ }^{\circ} \mathrm{C}$ e radiação de $1000 \mathrm{~W} / \mathrm{m}^{2}$, são apresentados na Tabela 1 . Os parâmetros do circuito Boost encontram-se na Tabela 2.

Tabela 1. Parâmetros dos módulos PV

\begin{tabular}{cc}
\hline Parâmetros & Valor \\
\hline \hline$V_{o c}$ & $32.9 \mathrm{~V}$ \\
$I_{s c}$ & $8.21 \mathrm{~A}$ \\
$\gamma$ & $3.18 \mathrm{~mA} /{ }^{\circ} \mathrm{C}$ \\
$\eta$ & 1.2 \\
$R_{s}$ & $5 \mathrm{~m} \Omega$ \\
$R_{p}$ & $7 \Omega$ \\
$N_{s}$ & 54 \\
\hline
\end{tabular}

Tabela 2. Parâmetros do conversor Boost

\begin{tabular}{cc}
\hline Parâmetros & Valor \\
\hline$C$ & $100 \mu \mathrm{F}$ \\
$L$ & $50 \mathrm{mH}$ \\
$R$ & $10 \mathrm{~m} \Omega$ \\
$V_{d c}$ & $350 \mathrm{~V}$ \\
\hline
\end{tabular}

Para análise da eficiência da técnica $\mathrm{P} \& \mathrm{O}$ tradicional, foram obtidas as curvas da energia perdida acumulada para diferentes valores da variação da corrente de referência $(\Delta I)$, partindo de $0.1 A$ até $1.0 A$, com incrementos de 0.1 A. Este resultado é apresentado na Figura 4.

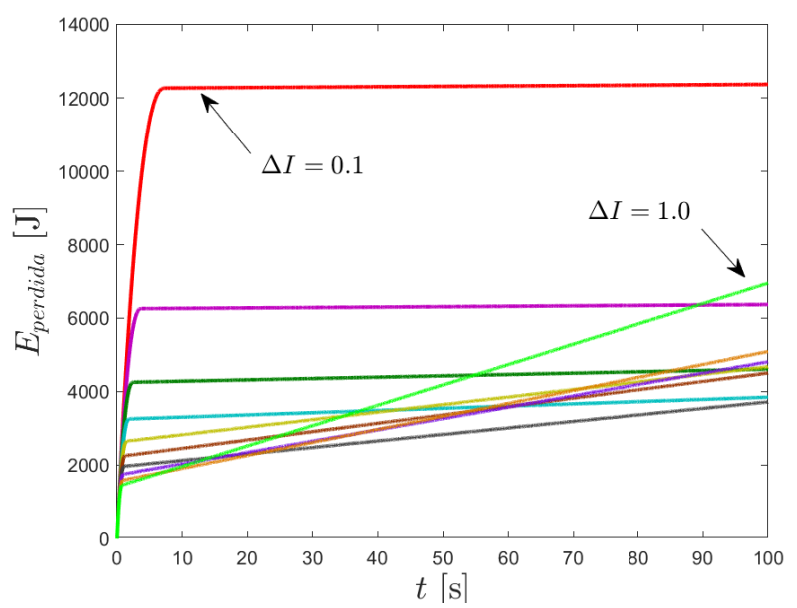

Figura 4. Curvas de energia perdida acumulada para diferentes valores de $\Delta I$.

A partir da Figura 4, percebe-se que a energia perdida apresenta um comportamento diferente de acordo com a variação de corrente imposta e nota-se que o tempo em que ocorre o ponto de inflexão (onde o método passa a oscilar em torno do MPP) também muda. Os pontos onde as diferentes curvas se cruzam caracteriza qual delas é melhor para transitórios e qual é melhor para regime dada uma certa frequência das variações das condições de operação do arranjo PV.

Pode-se destacar que quanto menor a variação de corrente (curva vermelha, $\Delta I=0,1 A$ ), maior a energia perdida no transitório. Entretanto, quanto maior a variação de corrente (curva verde claro, $\Delta I=1,0 A$ ), por mais que a energia perdida seja reduzida no transitório, esta continua crescendo consideravelmente após o ponto de inflexão.

O Exemplo 1 deixa claro que há um compromisso entre um bom transitório e um bom regime. A seção a seguir propõe uma forma de se evitar este compromisso através de uma adaptação em tempo real do algoritmo $\mathrm{P} \& \mathrm{O}$ tradicional, visando reduzir a energia perdida tanto em transitório quanto em regime.

\section{P\&O ADAPTÁVEL PROPOSTO}

Com base nos estudos e na verificação da possibilidade de melhoria do método $\mathrm{P} \& \mathrm{O}$ padrão (de $\Delta I$ fixo), propõese uma técnica que tem como princípio de funcionamento alterar o $\Delta I$ imposto pelo $\mathrm{P} \& \mathrm{O}$ a cada passo de operação. A ideia é que o valor de $\Delta I$ se adapte de acordo com a condição de operação do sistema, partindo de valores maiores para que o transitório seja rápido e reduzindo, quando é detectada a proximidade com o MPP, para um regime mais preciso. A estratégia é dividida em três algoritmos executados em sequência: (i) identificação automática da condição de $\mathrm{MPP}$, (ii) adaptação automática de $\Delta I$ e (iii) aplicação do $\mathrm{P} \& \mathrm{O}$ com variação adaptada.

A primeira função é apresentada no Algoritmo 2. O objetivo deste algoritmo é verificar se a operação está próxima 
ao máximo ponto de potência (MPP). Em caso positivo o algoritmo retorna uma flag $=1$. O funcionamento do código é detalhado na sequência.

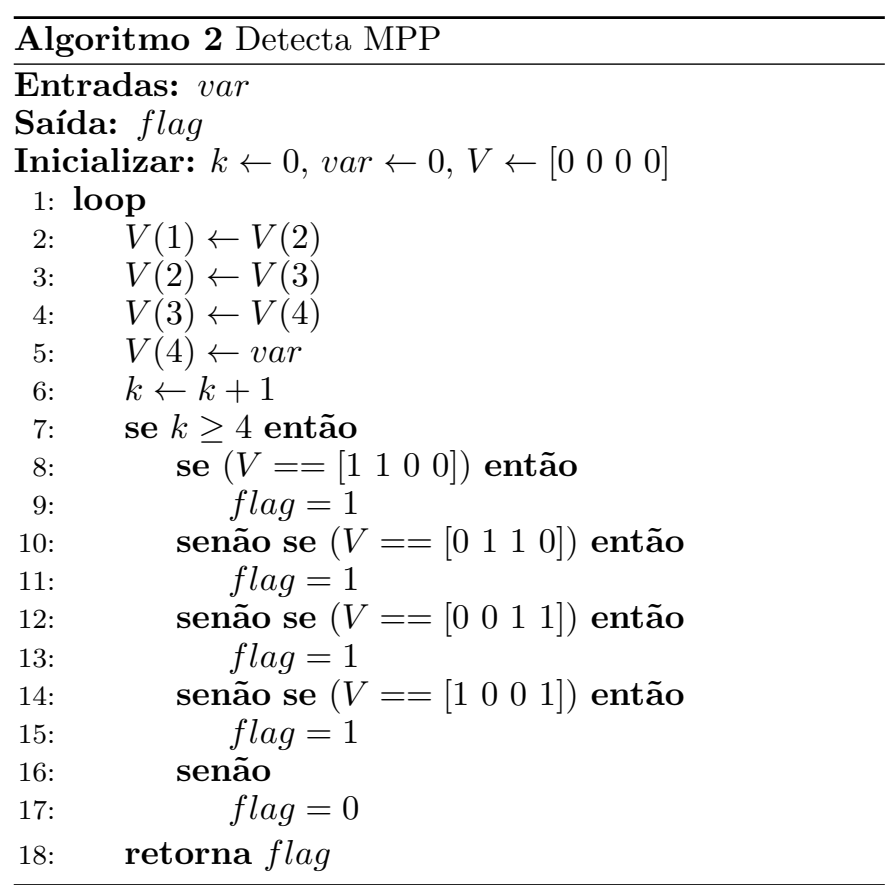

O algoritmo tem como base a identificação de padrões das direções de variação var, resultantes do Algoritmo 1. Para caracterizar que há oscilação em torno do MPP evitando falsas detecções, especialmente devido a mudanças bruscas de irradiação, são necessárias pelo menos quatro iterações do algoritmo P\&O. Por este motivo, define-se uma variável auxiliar $k$ para contar o número de iterações passadas desde que o algoritmo foi iniciado e assim que $k \geq 4$ pode-se julgar o padrão de comportamento. Para isso, o comportamento do da variação var das quatro últimas iterações ocorridas são armazenadas em um vetor $V$. Tal vetor opera como uma janela móvel, sempre armazenando o var (mais recente) no último elemento e movendo os mais antigos para as primeiras posições. Finalmente, comparase $V$ aos quatro padrões que caracterizam o MPP e, caso coincida com algum, a flag é setada.

Os padrões, apresentados nas Linhas 8, 10, 12 e 14, podem ser deduzidos analisando-se possíveis pontos de operação ao redor do MPP. Para ilustrar, considere a situação da Figura 5. Note que o máximo está entre os pontos de operação 1 e 2 , mas nesta ordem a potência continuou crescendo, não sendo detectado o MPP. Na iteração seguinte (entre 2 e 3), var muda de valor, mas seria errado afirmar que o MPP está neste intervalo. Como o var mudou, a operação retorna para o ponto 4 e apenas com uma última iteração de 4 para 5 pode-se afirmar, após detectar o padrão de var igual a [ $\left.\begin{array}{llll}1 & 1 & 0 & 0\end{array}\right]$, sabe-se onde está o MPP. Os demais padrões seguem um raciocínio análogo, mas com diferentes condições iniciais.

A condição de flag $=1$ habilita a próxima etapa do processo, o Algoritmo 3. Este é o estágio responsável pela determinação da alteração adaptativa na amplitude de variação da corrente de referência do perturba e observa.

No Algoritmo 3, inicialmente é verificado o valor da flag. Caso esta esteja setada, o máximo foi atingido e é iniciada

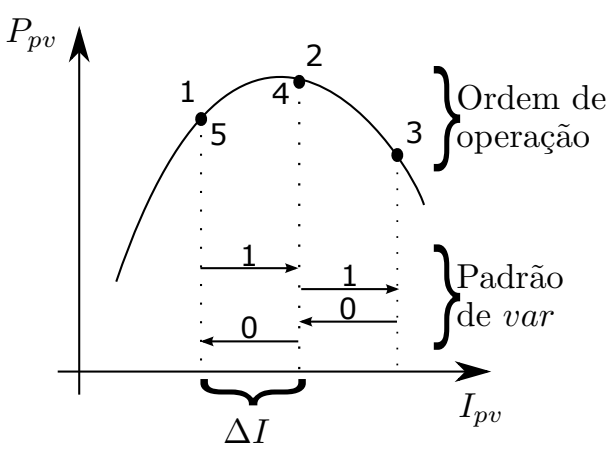

Figura 5. Padrão de var ao redor do MPP para uma dada condição inicial.

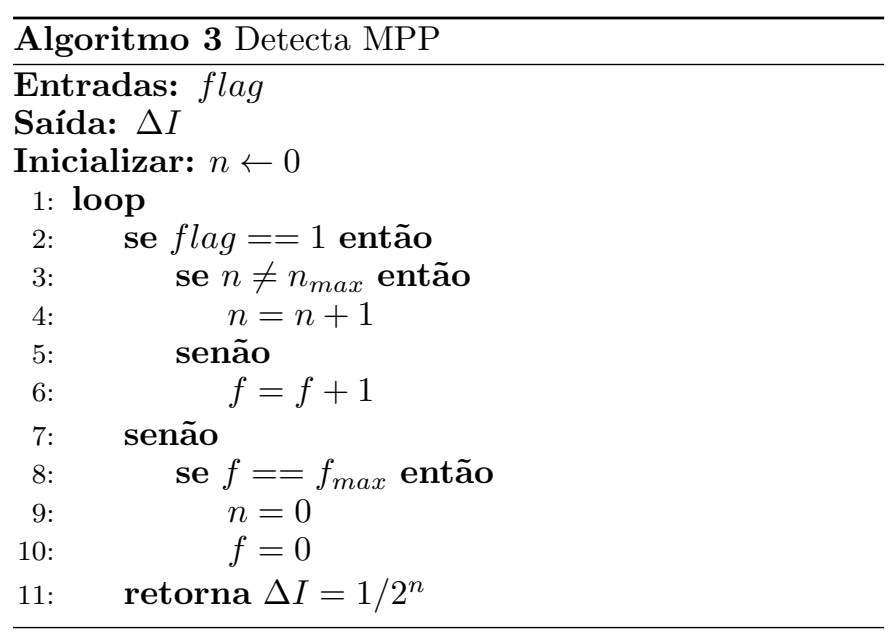

a redução da amplitude de $\Delta I$. Neste trabalho sugere-se que a adaptação seja realizada seguindo a razão áurea da fórmula a seguir.

$$
\Delta I=\frac{1}{2^{n}}
$$

onde $n$ é uma variável auxiliar iniciada em 0 , o que implica que não há alteração com relação ao valor inicial de $\Delta I$, e incrementada de uma unidade a cada vez que a flag for detectada. No algoritmo é recomendada a inclusão de um valor máximo $n_{\max }$ para $n$, pois caso a variação fique muito pequena, pequenas variações e ruídos levam a não identificação correta do MPP pelo método proposto no Algoritmo 2, levando a uma falsa percepção de que o sistema retornou à condição de transitório.

Foi acrescentado também no Algoritmo 3 o contador $f$, que tem a função de apenas redefinir $n$ para o valor 0 caso o sistema já estivesse na situação de regime no caso de uma $f l a g=0$. A ideia é evitar que uma falsa detecção apenas já redefina $n$, que seja detectada um número $f_{\text {max }}$ de vezes. Este valor, escolhido pelo projetista, que se for pequeno fica sujeito a falsas detecções ou oscilações momentâneas e se for grande demora a agir sob variações reais do MPP.

Com a atuação conjunta dos três algoritmos é definido o valor de $\Delta I$, atualizando-o de acordo com a condição de operação. A sequência completa de operação do $\mathrm{P} \& \mathrm{O}$ adaptável proposto é apresentada no diagrama de blocos da Figura 6. O bloco controlador (em vermelho), que tem como função executar a lei de chaveamento que resulta no seguimento da referência de $I_{p v}$, pode conter qualquer técnica que se aplique em conjunto com métodos $\mathrm{P} \& \mathrm{O}$. 


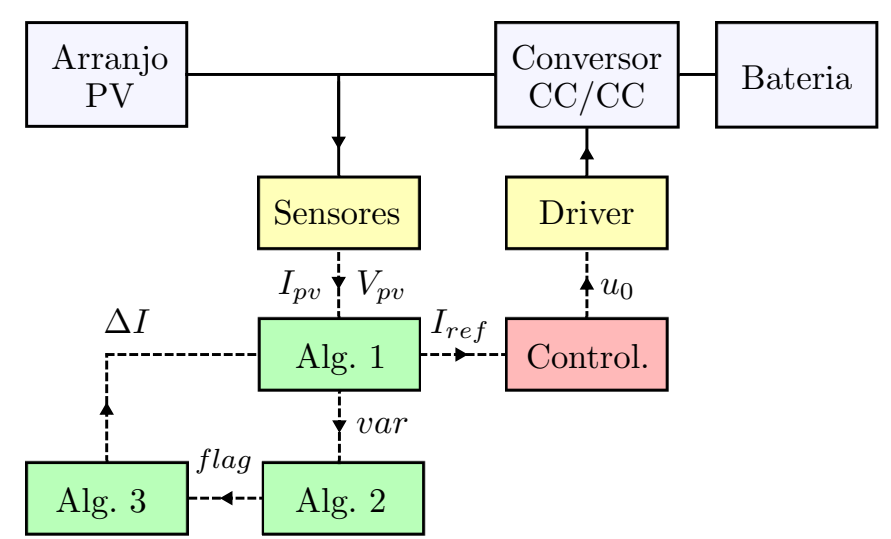

Figura 6. Diagrama de blocos da estrutura proposta.

\section{RESULTADOS E COMPARAÇÃO}

Esta seção apresenta resultados numéricos de simulação referentes à técnica proposta na Seção 4. São apresentadas também comparações com o MPPT P\&O tradicional. As simulações foram realizadas utilizando o software MatLab com o auxílio da ferramenta Simulink.

Exemplo 2. Considere o mesmo sistema apresentado no Exemplo 1. Serão considerados dois casos: (i) P\&O tradicional e (ii) $\mathrm{P} \& \mathrm{O}$ adaptável proposto. Para o caso (i) foi adotada uma variação fixa $\Delta I=0,5$, que é o valor médio dentre as análises do Exemplo 1, apresentando um compromisso razoável entre a energia perdida em transitório e em regime. Para o caso (ii) foi implementada a estrutura da Figura 6 e com a variação determinada pela Equação (7) inicialmente com $\Delta I=1$. É considerado também que $n_{\max }=3$, ou seja, o menor valor que $\Delta I$ atinge é 0,125 , e que $f_{\max }=2$.

Para ambos os casos foi determinada a energia perdida acumulada do sistema, durante um intervalo de 10 segundos, como pode ser visto na Figura 7 . Visando demonstrar a robustez do método perante variações em $T$ e $G$, estas variáveis foram subitamente alteradas seguindo o cronograma da Tabela 3. A última alteração ilustra um caso em que o $I_{p v}$ do MPP não varia significativamente, então espera-se uma não reação do algoritmo neste instante.

Tabela 3. Variações de radiação e temperatura.

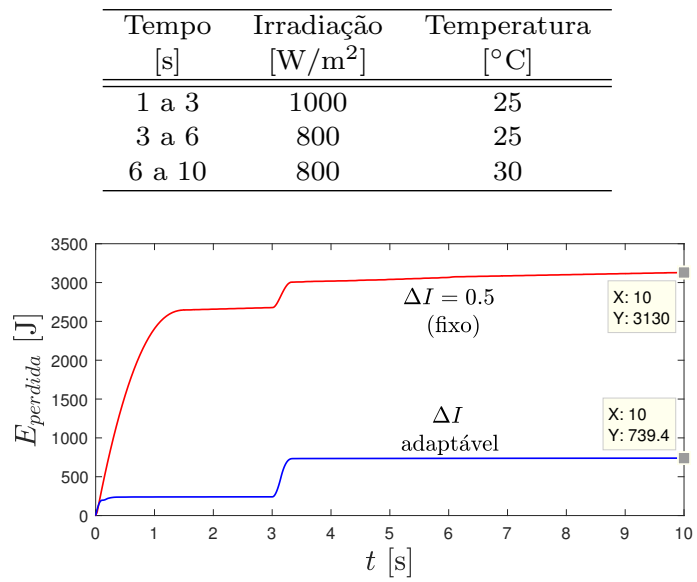

Figura 7. Energia acumulada perdida com a aplicação da técnica $\mathrm{P} \& \mathrm{O}$ tradicional.
A partir da Figura 7 é possível verificar que a energia perdida acumulada na técnica $\mathrm{P} \& \mathrm{O}$ tradicional chega a valores acima de $3130 \mathrm{~J}$ no período considerado, enquanto a técnica $\mathrm{P} \& O$ adaptável proposta não passa de $739.4 \mathrm{~J}$. Isso indica uma considerável redução da perda de energia e um consequente aumento da eficiência do sistema.

Pode-se perceber na Figura 4 que a técnica proposta atinge valores menores de energia perdida acumulada em comparação a qualquer passo fixo de variação analisado no Exemplo 1. Isso graças ao compromisso de se obter o melhor do transitório e o melhor do regime.

\section{CONCLUSÕES}

Este documento apresentou uma metodologia de análise da eficiência transitória e de regime de sistemas fotovoltaicos. Vale destacar a importância desta análise. Normalmente a eficiência de arranjos fotovoltaicos é avaliada de maneira estática, considerando apenas um dos dois regimes de operação do sistema.

Assim foi possível determinar numericamente as eficiências transitórias da técnica $\mathrm{P} \& \mathrm{O}$ tradicional de passo fixo, levando à identificação do compromisso entre um bom transitório e um regime eficiente. Em vista da problemática identificada, foi desenvolvido um algoritmo capaz de adaptar em tempo real a amplitude do passo do P\&O. Um exemplo numérico demonstrou a eficácia do algoritmo proposto, que resultou em uma significativa redução da energia perdida.

Dentre os trabalhos a serem desenvolvidos futuramente estão a determinação da melhor equação para definir a amplitude da variação da referência e a validação experimental dos resultados.

\section{REFERÊNCIAS}

Casaro, M.M. (2009). Inversor trifásico de dois estágios modificado aplicado no processamento da energia solar fotovoltaica em sistemas conectados à rede elétrica. Ph.D. thesis, Universidade Federal de Santa Catarina, Florianópolis, Brasil.

Dezuo, T.J.M., Trofino, A., and Scharlau, C.C. (2014). Switching rule design for sector-bounded nonlinear switched systems. In Proceedings of 19th International Federation of Automatic Control (IFAC) World Congress, 4074-4079. Cape Town, South Africa.

Di Francia, G. (2015). The effect of technology innovations on the cost of the photovoltaic electricity. In 4 th International Conference on Renewable Energy Research and Applications, 542-546. Palermo, Italy.

European Photovoltaic Industry Association (EPIA) (2018). Global market outlook for photovoltaics 20182022. Available in: http://www .epia.org.

Soualmia, A. and Chenni, R. (2016). A survey of maximum peak power tracking techniques used in photovoltaic power systems. IEEE Future Technologies Conference 2016, 430-443. 